\title{
Comments on "Patients' findings after COVID-19 infection and vaccinations: what to expect from $\left[{ }^{18} \mathrm{~F}\right] \mathrm{FDG} \mathrm{PET} / \mathrm{CT}$ "
}

\author{
Caroline Boursier $^{1} \cdot$ Pierre-Yves Marie ${ }^{1,2}$
}

Received: 12 January 2022 / Accepted: 23 January 2022 / Published online: 26 January 2022

(c) The Author(s), under exclusive licence to Springer-Verlag GmbH Germany, part of Springer Nature 2022

\section{Dear Sir,}

We read with great interest the editorial by Sollini et al. [1] about PET findings after COVID-19 infection and vaccination. As detailed in the editorial, the postvaccination FDG-PET imaging of cancer patients may provide an original insight regarding the magnitude of the immune-inflammatory response to the vaccine, with potential repercussions on the interpretation of PET images and more surprisingly, on the evolution of autoimmune diseases and cancer [1].

In most cases, this inflammatory response is localized to the site of injection and axillary lymph nodes. Signs of a systemic inflammatory response with increased FDG uptake by bone marrow and spleen, associated with an increase in various inflammation blood biomarkers, are much more unusual (see Table 1 in the editorial [1]). However, PET/CT imaging may also identify rare cases where the post-vaccine immune-inflammation response is not only intense but maladjusted, affecting organs that are generally not involved in the immune response, such as the myocardium.

Myocarditis appears to be the main significant, albeit very rare, complication of the novel COVID-19 messenger RNA (mRNA) vaccines [2]. For a still unknown reason, this type of myocarditis primarily affects young subjects a few days after the second mRNA-vaccine dose [2]. The mechanism by which myocarditis occurs early after the repeat injection

This article is part of the Topical Collection on Infection and inflammation

This comment refers to the article available online at https://doi. org/10.1007/s00259-021-05652-1.

Pierre-Yves Marie

py.marie@chru-nancy.fr

1 Department of Nuclear Medicine and Nancyclotep Imaging Platform, CHRU-Nancy Brabois, Université de Lorraine, Allée du Morvan, F-54000 Vandoeuvre-lès-Nancy, France

2 UMR 1116, INSERM, Université de Lorraine, F-54000 Nancy, France of mRNA vaccines needs to be elucidated, and PET tracers may be informative in this regard.

We recently published a case study in EJNMMI which reports post-mRNA vaccine myocarditis in two young male patients investigated by PET imaging of somatostatin receptors ( ${ }^{68} \mathrm{Ga}$-DOTATOC) [3]. Initial presentations at the acute phase were comparable to those of common infectious myocarditis with extended signs of inflammation on both PET and MRI images. This article was widely disseminated on social networks, particularly outside of the medical community (see the tweet report on https://link.altmetric.com/detai 1s/116892135), as this is the case for most articles dealing with COVID-19 vaccination including the present editorial from Sollini et al. [1]. Unfortunately, data presented in such articles may be misinterpreted and sometimes raises additional public concerns regarding vaccine safety, which is the most common reason cited for vaccine hesitancy. That is why we wish to seize the opportunity of this letter to provide an update on these two patients after their 4-month checkup performed as part of an ongoing clinical study (NCT03347760 on ClinicalTrials.gov). These checkups showed a complete resolution of the myocarditis, i.e., without any signs of inflammation on MRI and ${ }^{68} \mathrm{Ga}$-DOTATOC PET and without any left ventricular fibrosis or dysfunction on MRI. This further reinforces the rationale that myocarditis occurring after mRNA vaccination is not only very rare but is also associated with a mostly uncomplicated outcome. Moreover, it is also our responsibility, as physicians, to reiterate the evidence-based message that continuous COVID19 vaccination will help lower COVID-19 morbidity and mortality and far outweighs the rare cases of myocarditis and other rare adverse events that have been reported.

Finally, the editorial by Sollini et al. [1] also gives evidence of the magnitude of work required to move from clinical cases describing potential vaccination side effects to established cause-effect relationships, with potentially useful applications for understanding immune diseases and for cancer treatments. A large-scale collaborative international 
PET database is likely required to achieve this objective, given the scarcity of the vaccine side effects and the need to extract reliable scientific information.

Funding The reported myocarditis study (NCT03347760 on ClinicalTrials.gov) was funded by specific grants from the University Hospital of Nancy and the University of Lorraine (Clinical Research Contracts and Nancyclotep grants), with a free distribution of the DOTATOC kits from Novartis.

\section{Declarations}

Ethical approval and informed consent. All procedures reported in our two post-vaccine myocarditis patients were performed as part of an ongoing clinical study (NCT03347760 on ClinicalTrials.gov), in accordance with ethical standards and with the 1964 Helsinki declaration and its latest amendments. Approval was obtained from our Ethics Committee, and signed informed consent was obtained from these two patients.

Conflict of interest The authors declare no competing interests.

\section{References}

1. Sollini M, Gelardi F, Biroli M, Chiti A. Patients' findings after COVID-19 infection and vaccinations: what to expect from [18F] FDG PET/CT. Eur J Nucl Med Mol Imaging. 2021;20:1-5. https:// doi.org/10.1007/s00259-021-05652-1.

2. Klein NP, Lewis N, Goddard K, Fireman B, Zerbo O, Hanson KE, Donahue JG, Kharbanda EO, Naleway A, Nelson JC, Xu S, Yih WK, Glanz JM, Williams JTB, Hambidge SJ, Lewin BJ, Shimabukuro TT, DeStefano F, Weintraub ES. Surveillance for adverse events after COVID-19 mRNA vaccination. JAMA. 2021;326(14):1390-9. https://doi.org/10.1001/jama.2021.15072.

3. Boursier C, Chevalier E, Filippetti L, Imbert L, Roch V, Huttin O, Claudin M, Marie PY. ${ }^{68} \mathrm{Ga}$-DOTATOC digital-PET imaging of inflammatory cell infiltrates in myocarditis following COVID-19 vaccination. Eur J Nucl Med Mol Imaging. 2021;8:1-2. https:// doi.org/10.1007/s00259-021-05609-.

Publisher's note Springer Nature remains neutral with regard to jurisdictional claims in published maps and institutional affiliations. 